\title{
Accuracy of controlled attenuation parameter compared with ultrasound for detecting hepatic steatosis in children with severe obesity
}

\author{
Jurgen H. Runge ${ }^{1}$. Jet van Giessen ${ }^{1} \cdot$ Laura G. Draijer $^{2,3,4}$ - Eline E. Deurloo ${ }^{1}$ Anne M. J. B. Smets ${ }^{1}$. \\ Marc A. Benninga ${ }^{2} \cdot$ Bart G. P. Koot $^{2} \cdot$ Jaap Stoker ${ }^{1}$
}

Received: 29 April 2020 / Revised: 16 July 2020 / Accepted: 28 August 2020 / Published online: 10 September 2020

(C) The Author(s) 2020

\begin{abstract}
Objectives To determine the diagnostic accuracy of controlled attenuation parameter (CAP) on FibroScan ${ }^{\circledR}$ in detecting and grading steatosis in a screening setting and perform a head-to-head comparison with conventional B-mode ultrasound.

Methods Sixty children with severe obesity (median BMI z-score 3.37; median age 13.7 years) were evaluated. All underwent CAP and US using a standardized scoring system. Magnetic resonance spectroscopy proton density fat fraction (MRS-PDFF) was used as a reference standard.

Results Steatosis was present in 36/60 (60\%) children. The areas under the ROC (AUROC) of CAP for the detection of grade $\geq$ $\mathrm{S} 1, \geq \mathrm{S} 2$, and $\geq \mathrm{S} 3$ steatosis were 0.80 (95\% CI: 0.67-0.89), 0.77 (95\% CI: 0.65-0.87), and 0.79 (95\% CI: 0.66-0.88), respectively. The AUROC of US for the detection of grade $\geq$ S1 steatosis was 0.68 (95\% CI: 0.55-0.80) and not significantly different from that of CAP $(p=0.09)$. For detecting $\geq$ S1 steatosis, using the optimal cutoffs, CAP (277 dB/m) and US (US steatosis score $\geq 2$ ) had a sensitivity of $75 \%$ and $61 \%$ and a specificity of $75 \%$ and $71 \%$, respectively. When using echogenicity of liver parenchyma as only the scoring item, US had a sensitivity of $70 \%$ and specificity of $46 \%$ to detect $\geq$ S1 steatosis. The difference in specificity of CAP and US when using only echogenicity of liver parenchyma of $29 \%$ was significant $(p=0.04)$. Conclusion The overall performance of CAP is not significantly better than that of US in detecting steatosis in children with obesity, provided that the standardized scoring of US features is applied. When US is based on liver echogenicity only, CAP outperforms US in screening for any steatosis $(\geq \mathrm{S} 1)$.

Key Points

- The areas under the ROC curves of CAP and ultrasound (US) for detecting grade $\geq$ S1 steatosis were 0.80 and 0.68, respectively, and were not significantly different $(p=0.09)$.

- For detecting grade $\geq S 1$ steatosis in severely obese children, CAP had a sensitivity of $75 \%$ and a specificity of $75 \%$ at its optimal cutoff value of $277 \mathrm{~dB} / \mathrm{m}$.

- For detecting grade $\geq S 1$ steatosis in clinical practice, both CAP and US can be used, provided that the standardized scoring of US images is used.
\end{abstract}

Jurgen H. Runge and Jet van Giessen contributed equally to this work.

Jurgen H. Runge and Jet van Giessen shared first authorship.

Laura G. Draijer

1.g.draijer@amsterdamumc.nl

1 Department of Radiology and Nuclear Medicine, Amsterdam University Medical Centers, Location Academic Medical Center, University of Amsterdam, Amsterdam, The Netherlands

2 Department of Pediatric Gastroenterology and Nutrition, Amsterdam University Medical Centers, Location Academic Medical Center/ Emma Children's Hospital, University of Amsterdam, Amsterdam, The Netherlands
3 Amsterdam Reproduction \& Development Research Institute, Amsterdam University Medical Centers, Location Academic Medical Center/Emma Children's Hospital, Amsterdam, The Netherlands

4 Amsterdam UMC, University of Amsterdam, Gastroenterology and Hepatology, Amsterdam Gastroenterology Endocrinology Metabolism Research Institute, Amsterdam, The Netherlands 
Keywords Elasticity imaging techniques $\cdot$ Non-alcoholic fatty liver disease $\cdot$ Child $\cdot$ Obesity

\begin{tabular}{|c|c|}
\hline \multicolumn{2}{|c|}{ Abbreviations } \\
\hline ALT & Alanine aminotransferase \\
\hline AUROC & $\begin{array}{l}\text { Area under the receiver operating } \\
\text { characteristic (ROC) curve }\end{array}$ \\
\hline BMI & Body mass index \\
\hline CAP & Controlled attenuation parameter \\
\hline LR- & Negative likelihood ratio \\
\hline LR+ & Positive likelihood ratio \\
\hline MRS-PDFF & $\begin{array}{l}\text { Magnetic resonance spectroscopy } \\
\text { proton density fat fraction }\end{array}$ \\
\hline NPV & Negative predictive value \\
\hline PPV & Positive predictive value \\
\hline US score & Ultrasound steatosis score \\
\hline US & Ultrasound \\
\hline
\end{tabular}

\section{Introduction}

Due to the increasing prevalence of obesity, non-alcoholic fatty liver disease (NAFLD) has become the most common chronic liver disease in adults and children worldwide $[1,2]$. The NAFLD spectrum ranges from simple steatosis to nonalcoholic steatohepatitis (NASH), fibrosis, and cirrhosis. The pooled prevalence of NAFLD in children ranges from $7.6 \%$ in general population studies to $34.2 \%$ in studies based on child obesity clinics $[1,3,4]$. Although simple steatosis is the most common and benign stage in children, advanced fibrosis is reported in $17 \%$ of children with elevated alanine aminotransferase (ALT) referred from primary care to liver centers after screening [5]. In addition, NAFLD increases the risk of developing type 2 diabetes and cardiovascular diseases at adult age $[6,7]$. Timely detecting and staging of NAFLD is therefore of great importance, particularly in children who might be at a higher risk of complications in their lifetime given their longer life span [8-10].

Screening for NAFLD in children with obesity is propagated in most national and international obesity and hepatology guidelines [11]. However, the advised screening tools in these guidelines, serum ALT and conventional B-mode ultrasound (US), have a poor accuracy for detecting and grading steatosis [5, 12-20]. Magnetic resonance techniques, including magnetic resonance spectroscopy proton density fat fraction (MRS-PDFF), have excellent accuracy and reproducibility for detecting and grading liver steatosis in adults and children [21-26]. MRSPDFF has been accepted as a non-invasive reference standard for quantifying hepatic steatosis in studies [23, 27, 28]. These MR techniques are not suitable for screening in daily clinical practice due to relatively high costs and dependence on patient compliance, which can be a concern in the pediatric population. Therefore, an easy-to-use, inexpensive, and accurate screening tool for NAFLD is still very much needed.
In recent years, the controlled attenuation parameter (CAP) became available on the FibroScan ${ }^{\circledR}$ device (Echosens SA) for the detection of hepatic steatosis. CAP reflects the attenuation (loss of signal amplitude) of an ultrasound beam as it traverses tissue and is influenced by the amount of hepatic fat [29]. CAP uses standardized (controlled) settings, thereby minimizing user influence on the attenuation value. CAP is expressed as a number, whereas in conventional B-mode ultrasound, the grayscale values (which in part reflect the level of attenuation) are interpreted by an observer. These interpretations are subject to inter-observer variability. A second advantage of CAP is that FibroScan ${ }^{\circledR}$ simultaneously measures liver stiffness to assess fibrosis, which is of great benefit when evaluating children with NAFLD.

The aim of this study was to determine the diagnostic accuracy of CAP in (a) detecting and (b) grading hepatic steatosis in children with obesity in a screening setting using MRS-PDFF as the reference standard. As a secondary aim, we performed a head-to-head comparison of the accuracy of CAP and US in detecting steatosis in children with obesity.

\section{Methods}

\section{Study design and patients}

This prospective study was registered in the Dutch Trial Register (NL4155). Participants were recruited from the outpatient obesity clinics of two hospitals in The Netherlands (Slotervaart Ziekenhuis and Amsterdam UMC), during a 4year study period between 2014 and 2018. In The Netherlands, all children with obesity grade 3 ("severe obesity") or grade 1 or 2 obesity with elevated glucose levels are referred to obesity clinics for evaluation of metabolic complications. Inclusion criteria were age $8-18$ years and obesity (BMI z-score $>2$ ). The exclusion criteria were presence of a liver disease other than NAFLD (viral/autoimmune hepatitis, Wilson disease, hemochromatosis, alpha-1 antitrypsin deficiency), known focal liver lesion(s) in the right liver lobe (proven with histology results or imaging), use of steatogenic drugs, and contraindications for MRI. Patients were consecutively included. All participants underwent conventional Bmode US of the liver, CAP measurement on FibroScan ${ }^{\circledR}$, and MRS-PDFF at our tertiary hospital after a fasting period of at least 6 hours. The measurements were obtained during one or two visits, depending on the availability of MRI, FibroScan ${ }^{\circledR}$, and US. The study was approved by the Medical Ethics Committee of the Amsterdam UMC, location Academic Medical Center. Written informed consent was obtained from the participants aged 12 years or older and/or their 
Table 1 Scoring of hepatic steatosis with ultrasound (US steatosis score)

\begin{tabular}{ll}
\hline Score 0 & Normal echogenicity of liver parenchyma \\
& Normal visualization of diaphragm and intrahepatic blood vessels \\
& Slightly increased echogenicity of liver parenchyma \\
Score 1 & Normal visualization of diaphragm and intrahepatic blood vessels \\
& Markedly increased echogenicity of liver parenchyma \\
Score 2 & Slightly decreased visualization of diaphragm and intrahepatic blood vessels \\
& Severely increased echogenicity of liver parenchyma \\
Score 3 & No or severely decreased visualization of diaphragm and intrahepatic blood \\
& vessels and posterior part of the right liver lobe
\end{tabular}

legal guardians. We followed the Standards for Reporting of Diagnostic Accuracy (STARD) guidelines in this study on the accuracy of CAP and US in detecting hepatic steatosis [30].

\section{Sample size calculation}

Sample size calculations were performed for both primary and secondary outcomes; as the secondary outcome required a larger sample size, this is given here. Bohte et al previously showed that US had a sensitivity of $85 \%$ (95\% CI: 77-91) and a specificity of $55 \%$ (95\% CI: 46-65) for detecting any degree of steatosis in children with obesity [12]. Based on studies performed in adults, we hypothesized that CAP would have a specificity at least $15 \%$ higher than US. Using a McNemar test, we calculated that 26 non-steatotic subjects were required to reach a significant difference in specificity between US and CAP. Given the expected prevalence of steatosis (diagnosed on MRS-PDFF) of 50\% [12], a sample size of 52 subjects was anticipated required. After 40 subjects were included, interim analysis revealed a lower prevalence of non-steatotic subjects than anticipated (43\%). The number of required subjects was thereupon increased to 60 to allow more non-steatotic subjects to be included. Regardless of the final prevalence, study inclusion was stopped after 60 subjects had completed all study procedures.

\section{Clinical assessment}

Physical examination included measurement of weight, height, body mass index (BMI), waist circumference, and blood pressure. The BMI z-score was calculated. The BMI $\mathrm{Z}$-score is the number of standard deviations (SD) from the mean on a standard BMI curve for age and gender. Children with a BMI z-score of $>2$ (equals $>95$ th percentile) were considered to have obesity, and those with a BMI z-score of $>2.6$ (equals $>99$ th percentile) were considered to have severe obesity [31, 32].

\section{Laboratory tests}

Alanine aminotransferase (ALT) levels were obtained from the patients' clinical record. Values obtained within 3 months to the study visit were used for analysis. No blood was sampled for the purpose of this study.

Table 2 Patient characteristics

\begin{tabular}{|c|c|}
\hline \multicolumn{2}{|l|}{ Demographic } \\
\hline Age (years) & $13.7(12.1-16.1)$ \\
\hline Female, $n(\%)$ & $26(43)$ \\
\hline \multicolumn{2}{|l|}{ Ethnicity, $n(\%)$} \\
\hline Turkish & $23(38)$ \\
\hline Caucasian & $16(27)$ \\
\hline African & $4(7)$ \\
\hline Surinamese & $3(5)$ \\
\hline Asian & $3(5)$ \\
\hline North-African & $2(3)$ \\
\hline Other/unknown & $9(15)$ \\
\hline \multicolumn{2}{|l|}{ Clinical } \\
\hline Steatosis, $n(\%)$ & $36(60)$ \\
\hline BMI z-score & $3.37(3.01-3.98)$ \\
\hline Waist circumference, $\mathrm{cm}$ (IQR) & $100(90-121)$ \\
\hline \multicolumn{2}{|l|}{ Biological data } \\
\hline ALT, IU/L (IQR) & $28(19-39)$ \\
\hline \multicolumn{2}{|l|}{ MRS-PDFF } \\
\hline Fat fraction, \% (IQR) & $5.90(2.12-15.38)$ \\
\hline $\mathrm{S} 0, n(\%)$ & $24(40)$ \\
\hline $\mathrm{S} 1, n(\%)$ & $21(35)$ \\
\hline $\mathrm{S} 2, n(\%)$ & $7(12)$ \\
\hline $\mathrm{S} 3, n(\%)$ & $8(13)$ \\
\hline \multicolumn{2}{|l|}{ Ultrasound } \\
\hline US steatosis score $0, n(\%)$ & $16(27)$ \\
\hline US steatosis score $1, n(\%)$ & $15(25)$ \\
\hline US steatosis score $2, n(\%)$ & $16(27)$ \\
\hline US steatosis score $3, n(\%)$ & $13(21)$ \\
\hline
\end{tabular}

Continuous variables are expressed in median with interquartile range (IQR) in parentheses or $n(\%)$. ALT was measured in 40 patients. ALT alanine aminotransferase, $B M I$ body mass index, $M R S-P D F F$ magnetic resonance spectroscopy proton density fat fraction 


\section{Reference test: proton density fat fraction with magnetic resonance spectroscopy}

All subjects underwent an MRI scan after fasting, consisting of localizers and several anatomic imaging sequences followed by the MRS-PDFF sequence. Briefly, a single breath-hold, multi-echo stimulated echoes acquisition mode (STEAM) ${ }^{1} \mathrm{H}$-MRS sequence was performed using a $20 \times 20 \times 20 \mathrm{~mm}^{3}$ voxel positioned in segment VI or VII of the liver. Further details on acquisition and post-processing have been described in a previous study in our hospital. MRI technicians were blinded to clinical information and CAP and US results. Steatosis grades 1 (S1), S2, and S3 were defined as an MRS-PDFF fat fraction of $>4.14 \%,>15.72 \%$, and $>20.88 \%$, respectively. These thresholds were validated to correspond with steatosis grades 1, 2, and 3 of the NAFLD Activity Score (NAS) based on liver histology in adults in a previous study in our hospital [33].

\section{Index test 1: controlled attenuation parameter}

The CAP measurement was obtained using the FibroScan ${ }^{\circledR} 502$ Touch Device with the $3.5-\mathrm{MHz} \mathrm{M}$ probe. CAP was measured intercostally, in a midaxillary location, by one of two observers (J.R., a radiology resident who has performed more than 100 examinations, and B.K., a pediatric gastroenterologist who has performed more than 400 examinations). Both observers were blinded to US and MRS-PDFF results. Only CAP results with at least 10 valid measurements were analyzed.

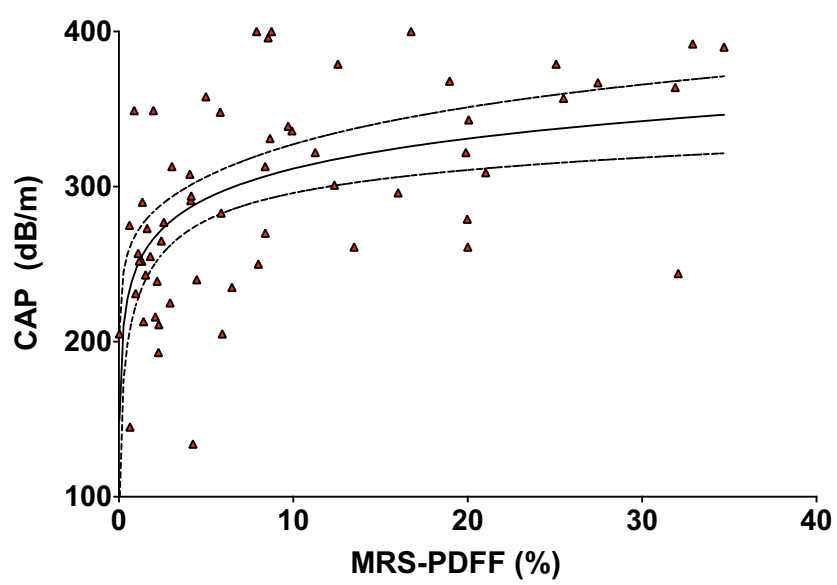

Fig. 1 Scatterplot of CAP by MRS-PDFF. Scatterplot shows logarithmic relationship between $\mathrm{CAP}(\mathrm{dB} / \mathrm{m})$ and $\operatorname{MRS}-\operatorname{PDFF}(\%)$. Solid and dashed lines represent lines of best fit with corresponding $95 \%$ confidence interval bands. $C A P$ controlled attenuation parameter, $M R S-P D F F$ magnetic resonance spectroscopy proton density fat fraction

\section{Index test 2: conventional B-mode ultrasound}

The US assessment was performed using the ATL HDI 5000 IU22 and Epiq 5G (Philips Healthcare) equipped with 5-2 and 9-2 curved-array transducers. US was performed by one of two pediatric radiologists with 10 and 30 years of experience in pediatric US. Both were blinded to any other result. The "abdominal general" setting was used and gain and focus were manually adjusted, depending on patient habitus and beam attenuation. The following standardized views of the liver were obtained: transverse and longitudinal views of the right hepatic lobe, including the right kidney and diaphragm; a sagittal view of the left liver lobe; a view including the portal vasculature; and a view of the gallbladder region. To assess steatosis, the following four widely accepted items were evaluated by the radiologists: echogenicity of liver parenchyma, visualization of diaphragm, visualization of intrahepatic vessels, and visualization of posterior part of the right hepatic lobe [34]. Ultrasound images of these features were previously shown by Bohte et al [12]. A final qualitative score from 0 to 3 was given by the radiologists with respect to liver steatosis: the "ultrasound steatosis score" (US steatosis score; Table 1) [12]. We calculated the optimal US steatosis score to separate grade S0 steatosis from S1-S3, S0-S1 from S2-S3, and S0-S2 using the Youden index that maximizes sensitivity and specificity. We applied these optimal US steatosis scores to determine the accuracy of US in detecting $\geq S 1, \geq S 2$, and $\geq S 3$. In clinical practice, steatosis is often assessed based only on increased liver echogenicity, without taking other features into account. Therefore, we additionally determined the accuracy of US when using echogenicity of liver parenchyma as only scoring item and compared this with CAP.

\section{Statistical analysis}

Descriptive statistics were used to analyze patients' demographic, laboratory, and imaging data. We calculated the optimal cutoff value for $\mathrm{CAP}$ to separate grade $\mathrm{S} 0$ steatosis from S1-S3, S0-S1 from S2-S3, and S0-S2 from S3 using the Youden index. The diagnostic accuracy of CAP and US for grading steatosis was calculated, including sensitivity, specificity, positive predictive value (PPV), negative predictive value (NPV), positive and negative likelihood ratios (LR+, LR-), and receiver operating characteristic (ROC) curve with 95\% confidence intervals. We compared the areas under the ROC curves (AUROC) of CAP and US using a (pairwise) comparison according to the method used by Hanley and McNeil [35]. We compared sensitivity and specificity of $\mathrm{CAP}$ and US in detecting $\geq \mathrm{S} 1$ steatosis using the McNemar chi-square test. A value of $p<0.05$ was considered a statistically significant difference. Statistical analyses were performed by using software (SPSS, version 22 [IBM]; GraphPad Prism 8.0 [GraphPad Software]; MedCalc 
Fig. 2 Box-and-whisker plot. Box-and-whisker plot of CAP according to steatosis grade at MRS-PDFF. Boxes show median and 25 th and 75 th percentiles, and whiskers show minimum and maximum values. Horizontal brackets indicate significant differences between steatosis grades. CAP controlled attenuation parameter,

MRS-PDFF magnetic resonance spectroscopy proton density fat fraction

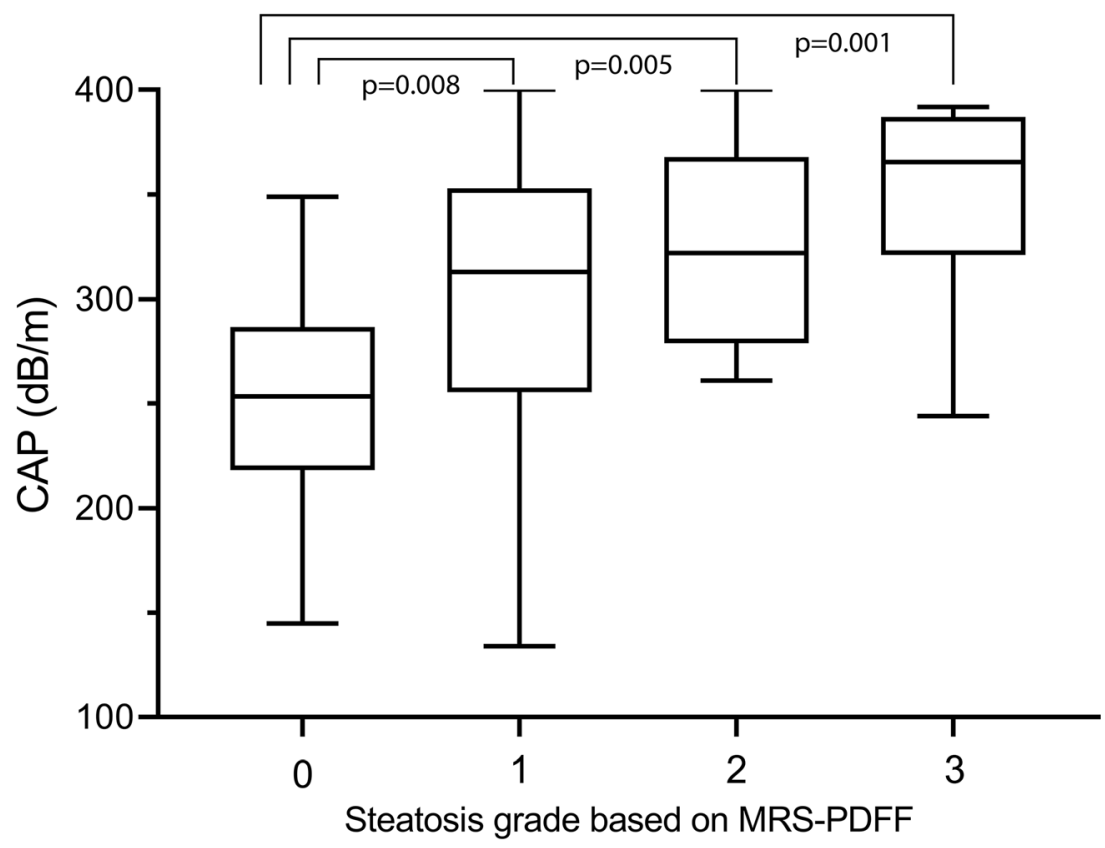

Statistical Software, version 16.2.0 [MedCalc Software; https://www.medcalc.org; 2016]).

\section{Results}

Sixty-two participants (34 males, 28 females) were consecutively included between October 2014 and December 2018. One participant refused MRI measurement and in one patient CAP measurement failed, most likely due to a large waist circumference. Patient characteristics are summarized in Table 2. The median age was 13.7 years (IQR 12.1-16.1). Median BMI z-score was 3.37 (IQR 3.01-3.98). Most participants were of Turkish or Dutch descent $(38 \%$ and $27 \%$, respectively). The median interval between MRS-PDFF and the index tests was 0 days (IQR $0-4$ ). Hepatic steatosis, defined as an MRS-PDFF fat fraction of $>4.14 \%$, was present in $36 / 60$ children $(60 \%)$. Grade 2 steatosis was found in 7 patients and 8 patients had grade 3 steatosis based on MRS-PDFF.

\section{CAP versus ultrasound}

CAP and US were successfully obtained in 60 patients. Median CAP value was $253 \mathrm{~dB} / \mathrm{m}$ (IQR 218-287) in children without steatosis versus $327 \mathrm{~dB} / \mathrm{m}$ (IQR 272-368) in children with steatosis. The scatterplot in Fig. 1 shows the logarithmic relationship between CAP and MRS-PDFF. Figure 2 shows
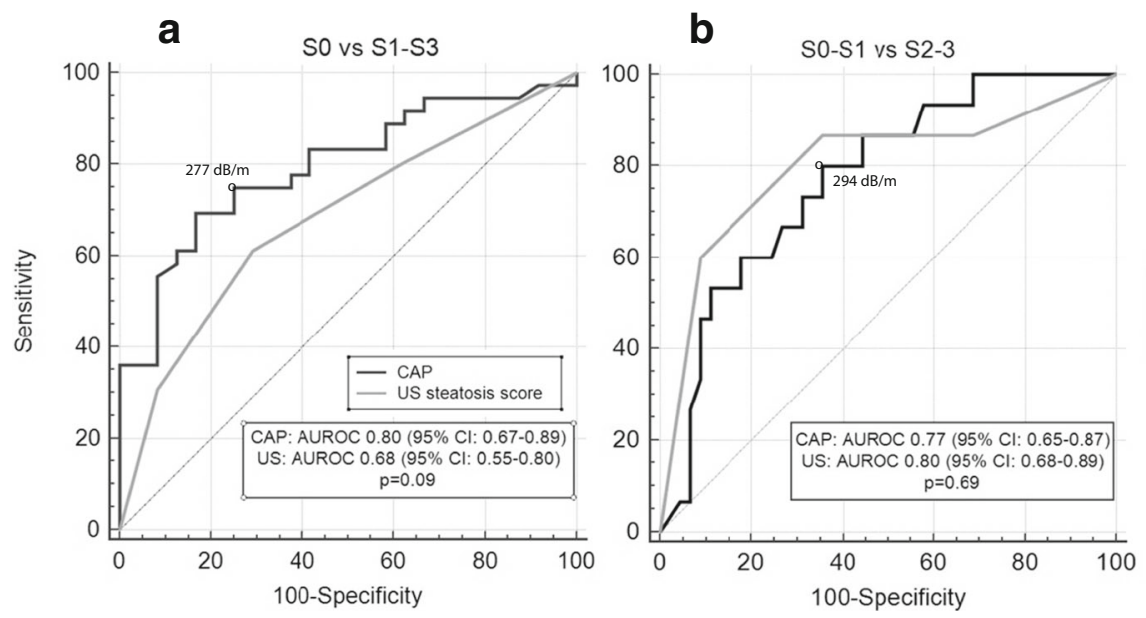

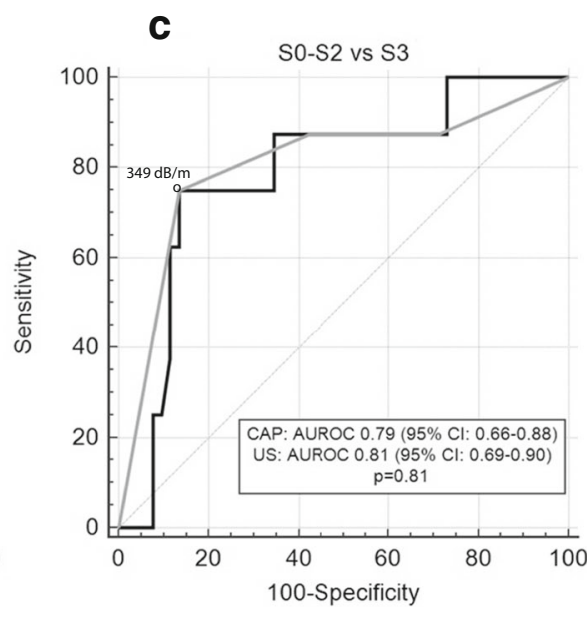

Fig. 3 Comparison of ROC curves. Comparison of ROC curves for three cutoffs. a Grade S0 steatosis versus grades $\mathrm{S} 1-\mathrm{S} 3$. b Grades $\mathrm{S} 0-\mathrm{S} 1$ versus S2-S3. c Grades S0-S2 versus S3. Black and gray lines indicate CAP and US, respectively. Optimal cutoffs are calculated using the Youden index. AUROC area under the ROC curve, CAP controlled attenuation parameter, ROC receiver operating curve, US ultrasound 


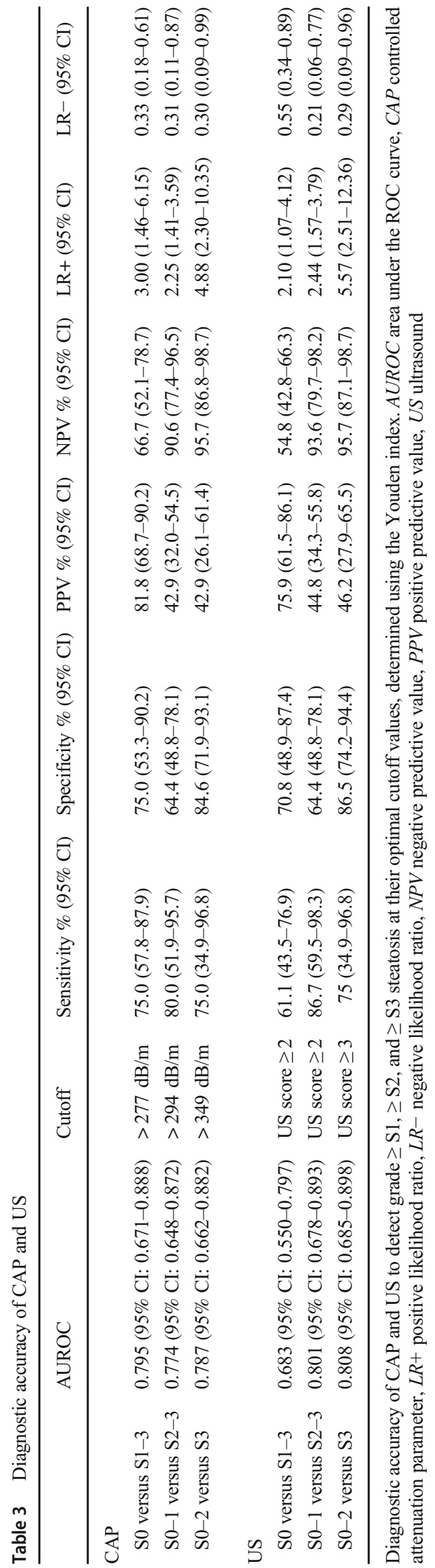

the distribution of CAP for the steatosis grades defined by MRS-PDFF. CAP differed significantly between grade S0 (no steatosis) and all other steatosis grades (S1 $p=0.008$; $\mathrm{S} 2$ $p=0.005$; S3 $p=0.001)$. CAP did not significantly differ between S1 and S2 $(p=0.54), \mathrm{S} 1$ and $\mathrm{S} 3(p=0.10)$, and $\mathrm{S} 2$ and $\mathrm{S} 3(p=0.36)$.

The AUROCs of CAP and US for the detection of grade $\geq$ $\mathrm{S} 1, \geq \mathrm{S} 2$, and $\geq \mathrm{S} 3$ steatosis were 0.80 versus $0.68,0.77$ versus 0.80 , and 0.79 versus 0.81 , respectively (Fig. 3 ). There was no significant difference in the AUROC of CAP and US for the detection of any stage of steatosis ( $\mathrm{S} 0 \mathrm{vs} \geq \mathrm{S} 1, p=0.09$ ) or the higher grades of steatosis.

Based on the Youden index, the optimal cutoff value for CAP for detecting $\geq \mathrm{S} 1$ steatosis was $277 \mathrm{~dB} / \mathrm{m}$, yielding a sensitivity of $75 \%$ and a specificity of $75 \%$ (Table 3 ). At its optimal cutoff value (US score $\geq 2$ ), US had a sensitivity of $61 \%$ and a specificity of $71 \%$ for detecting $\geq \mathrm{S} 1$ steatosis (Table 3 ). The difference in sensitivity between CAP and US of $14 \%$ did not reach statistical significance $(p=0.23)$. When using echogenicity of liver parenchyma as only the scoring item, US had a sensitivity of $70 \%$ and specificity of $46 \%$ to detect $\geq$ S1 steatosis. The difference in specificity of CAP and US when using only echogenicity of liver parenchyma of $29 \%$ was significant $(p=0.04)$.

The optimal diagnostic accuracies of CAP and US to detect different stages $(\geq \mathrm{S} 1, \geq \mathrm{S} 2, \geq \mathrm{S} 3)$ of steatosis using their optimal cutoffs are shown in Table 3.

\section{Discussion}

This study reports on the accuracy of CAP and conventional B-mode US for detecting steatosis in children with obesity using MRS-PDFF as the reference standard. This is the first head-to-head comparison of CAP and US in children with obesity. The overall performance of CAP was not significantly better than that of US.

When screening for NAFLD, detecting steatosis is usually the first step to identify those patients that require additional testing to stage NAFLD, i.e., detecting and grading inflammation and/or fibrosis. The most widely used screening tools, serum ALT measurement and ultrasound, are limited by their mediocre accuracy which has led to the development of new techniques such as MRI-based measurements and CAP, an ultrasound-based measurement available on the FibroScan ${ }^{\circledR}$ device. To evaluate the clinical value of new screening tools for NAFLD, it is most relevant to determine the accuracy of detecting any steatosis $(\geq \mathrm{S} 1)$, since the degree of steatosis is not associated with the histological severity of liver disease and metabolic changes [36, 37]. For example, insulin resistance starts early on after hepatic triglyceride accumulation and is fully established at $1.5 \%$ (measured by ${ }^{1} \mathrm{H}-\mathrm{MRS}$ ), 
which is well below the current cutoff point for the diagnosis of NAFLD [37].

Comparing the accuracy data from this study to those from previous studies is difficult as study populations and reference standards differ among studies. The performance of CAP for detecting $\geq \mathrm{S} 1$ steatosis, reflected in the area under the ROC curve $(0.80 ; 95 \%$ CI: $0.67-0.89)$, is comparable to that of adult studies: 0.85 in a meta-analysis in 2014 [38] and $0.77-$ 0.96 in recent studies [33, 39-44]. In comparison to pediatric studies, Ferraioli et al found an AUROC of 0.84 (95\% CI: $0.78-0.89)$ to detect $\geq \mathrm{S} 1$ steatosis in a mixed population of children with and without obesity [45]. However, US was used as the reference standard which is less accurate than MRS-PDFF. Desai et al reported a higher performance of CAP: AUROC of 0.93 (95\% CI: 0.87-0.99) and a sensitivity of $87 \%$ and specificity of $83 \%$ in detecting $\geq \mathrm{S} 1$ steatosis at the optimal cutoff point, using biopsy as the reference standard [46]. This difference may be accounted to the difference in study population, as they included children with various liver diseases; only 14 out of 69 had NAFLD, and mean BMI zscore was 0.67 compared with a median BMI z-score of 3.51 in our study. Shin et al evaluated 86 children with and without obesity and also reported a higher accuracy of CAP: AUROC 0.94 (95\% CI: $0.87-0.98$ ) and a sensitivity of $99 \%$ and specificity of $80 \%$ in detecting $\geq \mathrm{S} 1$ steatosis using MRI-PDFF as the reference standard [47]. Again the difference in BMI might explain the difference in performance, since only $17 /$ 83 children had severe obesity versus all children in our study. In agreement with our results, they found that discrimination between S1, S2, and S3 steatosis was suboptimal.

Thus far, different optimal CAP thresholds have been found in studies. In our study, a threshold of $277 \mathrm{~dB} / \mathrm{m}$ was found to be most optimal to detect $\geq \mathrm{S} 1$ steatosis which is comparable to the proposed thresholds from adult studies that range between $236 \mathrm{~dB} / \mathrm{m}$ and $302 \mathrm{~dB} / \mathrm{m}[33,39,40,42,43$, 48]. This threshold is higher compared with the pediatric studies by Desai et al [46] and Shin et al [47]: $225 \mathrm{~dB} / \mathrm{m}$ and $241 \mathrm{~dB} / \mathrm{m}$, respectively. The latter could be explained by the different study populations and underlying disorders, since in adults CAP values have been found to be significantly associated with BMI, waist circumference, and the studied liver disease, including higher values in NAFLD [41]. This implies that disease- or patient-group-specific thresholds are required. The threshold determined in this study needs validation in another population-based cohort of children with obesity.

To ensure a fair comparison between the accuracy of CAP (continuous variable) and US (categorical variable) in detecting $\geq \mathrm{S} 1$ steatosis, we used the optimal thresholds for the detection of $\geq$ S1 steatosis of both tools. The US threshold in this study (US score $\geq 2$ ) is in line with the previous reported pooled accuracy results of US [11]. In our study, CAP had a $14 \%$ higher sensitivity compared with US. This difference was not significant as our study was powered on detecting a difference of $15 \%$ in specificity in a cohort with lower anticipated prevalence of steatosis. To determine whether there is a small difference in accuracy between CAP and US, a larger study is needed. For clinical practice, this study implies that both CAP and US can be used for screening purposes depending on local availability and expertise.

When using ultrasound in clinical practice, steatosis is often considered present when only liver echogenicity is increased, and other US features (e.g., decreased visibility of intrahepatic vessels, decreased visualization of the diaphragm) are less frequently used or specifically mentioned in radiological reports. We found that when taking only liver echogenicity into account, specificity of CAP was $29 \%$ higher $(p=0.04)$. These findings underscore the relevance of evaluating more than just the increased echogenicity of the liver parenchyma when using US in children suspected to have steatosis.

The strength of this study is the use of MRS-PDFF as the reference standard as it has high accuracy in detecting and grading steatosis. In addition, the MRS-PDFF setting used in this study has been validated compared with histology [33]. Validation was performed in adults since validation with histology in a pediatric cohort of NAFLD is unfeasible due to ethical objections. Another strength is the inclusion of a multiethnic study population which is representative of the population targeted for NAFLD screening. This study therefore evaluates the usefulness of CAP and US in a setting identical to the real-life screening situation. A limitation of the current study is its sample size and the low number of children with higher steatosis grades, hampering the comparison of accuracy of CAP and US in grading steatosis. Also, we were not able to correlate CAP with metabolic factors, as no blood was drawn for the purpose of this study.

In conclusion, this study shows that CAP has a sensitivity and a specificity of both $75 \%$ at the optimal threshold of $277 \mathrm{~dB} / \mathrm{m}$. However, the overall performance of CAP compared with US for detecting steatosis in children with obesity is not significantly better, provided that the standardized scoring of US images is used. When US is based on liver echogenicity only, CAP had a $29 \%$ higher specificity and thereby outperforms US in detecting steatosis.

Funding The authors state that this work has not received any funding.

\section{Compliance with ethical standards}

Guarantor The scientific guarantor of this publication is Jaap Stoker.

Conflict of interest The authors of this manuscript declare no relationships with any companies whose products or services may be related to the subject matter of the article.

Statistics and biometry Several authors have significant statistical expertise. Therefore, statistical advice was not needed for this manuscript. 
Informed consent Written informed consent was obtained from all subjects (patients) and legal guardians in this study.

Ethical approval Institutional Review Board approval was obtained.

\author{
Methodology \\ - prospective \\ - diagnostic study \\ - performed at one institution
}

Open Access This article is licensed under a Creative Commons Attribution 4.0 International License, which permits use, sharing, adaptation, distribution and reproduction in any medium or format, as long as you give appropriate credit to the original author(s) and the source, provide a link to the Creative Commons licence, and indicate if changes were made. The images or other third party material in this article are included in the article's Creative Commons licence, unless indicated otherwise in a credit line to the material. If material is not included in the article's Creative Commons licence and your intended use is not permitted by statutory regulation or exceeds the permitted use, you will need to obtain permission directly from the copyright holder. To view a copy of this licence, visit http://creativecommons.org/licenses/by/4.0/.

\section{References}

1. Wong RJ, Aguilar M, Cheung R et al (2015) Nonalcoholic steatohepatitis is the second leading etiology of liver disease among adults awaiting liver transplantation in the United States. Gastroenterology 148:547-555

2. Nobili V, Reale A, Alisi A et al (2009) Elevated serum ALT in children presenting to the emergency unit: relationship with NAFLD. Dig Liver Dis 41:749-752

3. Anderson EL, Howe LD, Jones HE, Higgins JP, Lawlor DA, Fraser A (2015) The prevalence of non-alcoholic fatty liver disease in children and adolescents: a systematic review and meta-analysis. PLoS One 10:e140908

4. Vajro P, Lenta S, Socha P et al (2012) Diagnosis of nonalcoholic fatty liver disease in children and adolescents: position paper of the ESPGHAN Hepatology Committee. J Pediatr Gastroenterol Nutr 54:700-713

5. Schwimmer JB, Newton KP, Awai HI et al (2013) Paediatric gastroenterology evaluation of overweight and obese children referred from primary care for suspected non-alcoholic fatty liver disease. Aliment Pharmacol Ther 38:1267-1277

6. Hecht L, Weiss R (2014) Nonalcoholic fatty liver disease and type 2 diabetes in obese children. Curr Diab Rep 14:448

7. Armstrong MJ, Adams LA, Canbay A, Syn WK (2014) Extrahepatic complications of nonalcoholic fatty liver disease. Hepatology 59:1174-1197

8. Feldstein AE, Charatcharoenwitthaya P, Treeprasertsuk S, Benson JT, Enders FB, Angulo P (2009) The natural history of nonalcoholic fatty liver disease in children: a follow-up study for up to 20 years. Gut $58: 1538-1544$

9. Molleston JP, White F, Teckman J, Fitzgerald JF (2002) Obese children with steatohepatitis can develop cirrhosis in childhood. Am J Gastroenterol 97:2460-2462

10. Alkhouri N, Hanouneh IA, Zein NN et al (2016) Liver transplantation for nonalcoholic steatohepatitis in young patients. Transpl Int 29:418-424

11. Koot BGP, Nobili V (2017) Screening for non-alcoholic fatty liver disease in children: do guidelines provide enough guidance? Obes Rev. https://doi.org/10.1111/obr.12556
12. Bohte AE, Koot BG, van der Baan-Slootweg OH et al (2011) US cannot be used to predict the presence or severity of hepatic steatosis in severely obese adolescents. Radiology 262(1):327-334

13. Pacifico L, Celestre M, Anania C, Paolantonio P, Chiesa C, Laghi A (2007) MRI and ultrasound for hepatic fat quantification:relationships to clinical and metabolic characteristics of pediatric nonalcoholic fatty liver disease. Acta Paediatr 96:542-547

14. Pozzato C, Radaelli G, Dall'Asta C et al (2008) MRI in identifying hepatic steatosis in obese children and relation to ultrasonography and metabolic findings. J Pediatr Gastroenterol Nutr 47:493-499

15. Shannon A, Alkhouri N, Carter-Kent C et al (2011) Ultrasonographic quantitative estimation of hepatic steatosis in children with NAFLD. J Pediatr Gastroenterol Nutr 53:190-195

16. Radetti G, Kleon W, Stuefer J, Pittschieler K (2006) Non-alcoholic fatty liver disease in obese children evaluated by magnetic resonance imaging. Acta Paediatr 95:833-837

17. Burgert TS, Taksali SE, Dziura J et al (2006) Alanine aminotransferase levels and fatty liver in childhood obesity: associations with insulin resistance, adiponectin, and visceral fat. J Clin Endocrinol Metab 91:4287-4294

18. Rehm JL, Connor EL, Wolfgram PM, Eickhoff JC, Reeder SB, Allen DB (2014) Predicting hepatic steatosis in a racially and ethnically diverse cohort of adolescent girls. J Pediatr 165:319325.e311

19. Molleston JP, Schwimmer JB, Yates KP et al (2014) Histological abnormalities in children with nonalcoholic fatty liver disease and normal or mildly elevated alanine aminotransferase levels. J Pediatr 164:707-713 e703

20. Draijer LG, Feddouli S, Bohte AE et al (2019) Comparison of diagnostic accuracy of screening tests ALT and ultrasound for pediatric non-alcoholic fatty liver disease. Eur J Pediatr. https://doi. org/10.1007/s00431-019-03362-3

21. Bohte AE, van Werven JR, Bipat S, Stoker J (2011) The diagnostic accuracy of US, CT, MRI and 1H-MRS for the evaluation of hepatic steatosis compared with liver biopsy: a meta-analysis. Eur Radiol 21:87-97

22. Awai HI, Newton KP, Sirlin CB, Behling C, Schwimmer JB (2014) Evidence and recommendations for imaging liver fat in children, based on systematic review. Clin Gastroenterol Hepatol 12:765773

23. Di Martino M, Pacifico L, Bezzi M et al (2016) Comparison of magnetic resonance spectroscopy, proton density fat fraction and histological analysis in the quantification of liver steatosis in children and adolescents. World J Gastroenterol 22:8812-8819

24. Schwimmer JB, Middleton MS, Behling C et al (2015) Magnetic resonance imaging and liver histology as biomarkers of hepatic steatosis in children with nonalcoholic fatty liver disease. Hepatology 61:1887-1895

25. Middleton MS, Van Natta ML, Heba ER et al (2018) Diagnostic accuracy of magnetic resonance imaging hepatic proton density fat fraction in pediatric nonalcoholic fatty liver disease. Hepatology 67: 858-872

26. Rehm JL, Wolfgram PM, Hernando D, Eickhoff JC, Allen DB, Reeder SB (2015) Proton density fat-fraction is an accurate biomarker of hepatic steatosis in adolescent girls and young women. Eur Radiol 25:2921-2930

27. Noureddin M, Lam J, Peterson MR et al (2013) Utility of magnetic resonance imaging versus histology for quantifying changes in liver fat in nonalcoholic fatty liver disease trials. Hepatology 58:19301940

28. European Association for the Study of the Liver (EASL); European Association for the Study of Diabetes (EASD); European Association for the Study of Obesity (EASO) (2016) EASLEASD-EASO clinical practice guidelines for the management of non-alcoholic fatty liver disease. J Hepatol 64:1388-1402 
29. Sasso M, Miette V, Sandrin L, Beaugrand M (2012) The controlled attenuation parameter (CAP): a novel tool for the non-invasive evaluation of steatosis using Fibroscan. Clin Res Hepatol Gastroenterol 36:13-20

30. Bossuyt PM, Reitsma JB, Bruns DE et al (2015) STARD 2015: an updated list of essential items for reporting diagnostic accuracy studies. BMJ 351:h5527

31. Cole TJ, Bellizzi MC, Flegal KM, Dietz WH (2000) Establishing a standard definition for child overweight and obesity worldwide: international survey. BMJ 320:1240-1243

32. Fredriks AM, van Buuren S, Wit JM, Verloove-Vanhorick SP (2000) Body index measurements in 1996-7 compared with 1980. Arch Dis Child 82:107-112

33. Runge JH, Smits LP, Verheij J et al (2018) MR spectroscopyderived proton density fat fraction is superior to controlled attenuation parameter for detecting and grading hepatic steatosis. Radiology 286:547-556

34. Saverymuttu SH, Joseph AE, Maxwell JD (1986) Ultrasound scanning in the detection of hepatic fibrosis and steatosis. Br Med J (Clin Res Ed) 292:13-15

35. Hanley JA, McNeil BJ (1983) A method of comparing the areas under receiver operating characteristic curves derived from the same cases. Radiology 148:839-843

36. Monteiro JM, Monteiro GM, Caroli-Bottino A, Pannain VL (2014) Nonalcoholic fatty liver disease: different classifications concordance and relationship between degrees of morphological features and spectrum of the disease. Anal Cell Pathol (Amst) 2014:526979

37. Bril F, Barb D, Portillo-Sanchez P et al (2017) Metabolic and histological implications of intrahepatic triglyceride content in nonalcoholic fatty liver disease. Hepatology 65:1132-1144

38. Shi KQ, Tang JZ, Zhu XL et al (2014) Controlled attenuation parameter for the detection of steatosis severity in chronic liver disease: a meta-analysis of diagnostic accuracy. J Gastroenterol Hepatol 29:1149-1158

39. Eddowes PJ, Sasso M, Allison M et al (2019) Accuracy of FibroScan controlled attenuation parameter and liver stiffness measurement in assessing steatosis and fibrosis in patients with nonalcoholic fatty liver disease. Gastroenterology 156:1717-1730

40. Imajo K, Kessoku T, Honda $\mathrm{Y}$ et al (2016) Magnetic resonance imaging more accurately classifies Steatosis and fibrosis in patients with nonalcoholic fatty liver disease than transient elastography. Gastroenterology 150:626-637.e627

41. de Ledinghen V, Vergniol J, Capdepont M et al (2014) Controlled attenuation parameter (CAP) for the diagnosis of steatosis: a prospective study of 5323 examinations. J Hepatol 60:1026-1031

42. Baumeler S, Jochum W, Neuweiler J, Bergamin I, Semela D (2019) Controlled attenuation parameter for the assessment of liver steatosis in comparison with liver histology: a single-centre real life experience. Swiss Med Wkly 149:w20077

43. Caussy C, Alquiraish MH, Nguyen P et al (2018) Optimal threshold of controlled attenuation parameter with MRI-PDFF as the gold standard for the detection of hepatic steatosis. Hepatology 67: 1348-1359

44. Pu K, Wang Y, Bai S et al (2019) Diagnostic accuracy of controlled attenuation parameter (CAP) as a non-invasive test for steatosis in suspected non-alcoholic fatty liver disease: a systematic review and meta-analysis. BMC Gastroenterol 19:51

45. Ferraioli G, Calcaterra V, Lissandrin R et al (2017) Noninvasive assessment of liver steatosis in children: the clinical value of controlled attenuation parameter. BMC Gastroenterol 17:61

46. Desai NK, Harney S, Raza R et al (2016) Comparison of controlled attenuation parameter and liver biopsy to assess hepatic steatosis in pediatric patients. J Pediatr 173:160-164.e161

47. Shin J, Kim MJ, Shin HJ et al (2019) Quick assessment with controlled attenuation parameter for hepatic steatosis in children based on MRI-PDFF as the gold standard. BMC Pediatr 19:112

48. Karlas T, Petroff D, Sasso M et al (2017) Individual patient data meta-analysis of controlled attenuation parameter (CAP) technology for assessing steatosis. J Hepatol 66:1022-1030

Publisher's note Springer Nature remains neutral with regard to jurisdictional claims in published maps and institutional affiliations. 